\title{
Chest CT for rapid triage of patients in multiple emergency departments during COVID-19 epidemic: experience report from a large French university hospital
}

\author{
Victoria Ducray $^{1}$ • Anna Sesilia Vlachomitrou ${ }^{2}$ - Maude Bouscambert-Duchamp ${ }^{3,4} \cdot$ Salim Si-Mohamed $^{1,5,6}$ (DD . $^{3}$

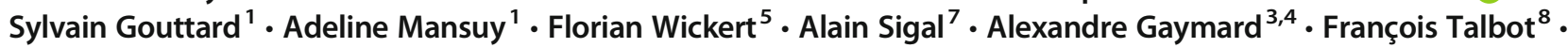 \\ Catherine Michel $^{8}$ • Thomas Perpoint ${ }^{9}$ • Jean-Baptiste Pialat ${ }^{1,5}$ • Olivier Rouviere ${ }^{1,10} \cdot$ Laurent Milot $^{1}$. \\ François Cotton $^{1,5} \cdot$ Philippe Douek $^{1,5}$ - Muriel Rabilloud ${ }^{11} \cdot$ Loic Boussel $^{1,5} \cdot$ On behalf of COVID-Outcomes-HCL \\ Consortium
}

Received: 10 June 2020 / Revised: 23 July 2020 / Accepted: 5 August 2020 / Published online: 19 August 2020

(C) European Society of Radiology 2020

\begin{abstract}
Objectives To assess the diagnostic performances of chest CT for triage of patients in multiple emergency departments during COVID-19 epidemic, in comparison with reverse transcription polymerase chain reaction (RT-PCR) test.

Method From March 3 to April 4, 2020, 694 consecutive patients from three emergency departments of a large university hospital, for which a hospitalization was planned whatever the reasons, i.e., COVID- or non-COVID-related, underwent a chest CT and one or several RT-PCR tests. Chest CTs were rated as "Surely COVID+," "Possible COVID+," or "COVID-" by experienced radiologists. Sensitivity, specificity, positive predictive value (PPV), and negative predictive value (NPV) were calculated using the final RT-PCR test as standard of reference. The delays for CT reports and RT-PCR results were recorded and compared.

Results Among the 694 patients, 287 were positive on the final RT-PCR exam. Concerning the 694 chest CT, 308 were rated as "Surely COVID+", 34 as "Possible COVID+," and 352 as "COVID-." When considering only the "Surely COVID+" CT as positive, accuracy, sensitivity, specificity, PPV, and NPV reached 88.9\%, 90.2\%, 88\%, 84.1\%, and 92.7\%, respectively, with respect to final RT-PCR test. The mean delay for CT reports was three times shorter than for RT-PCR results $(187 \pm 148$ min versus $573 \pm 327 \mathrm{~min}, p<0.0001)$.

Conclusion During COVID-19 epidemic phase, chest CT is a rapid and most probably an adequately reliable tool to refer patients requiring hospitalization to the COVID+ or COVID- hospital units, when response times for virological tests are too long.

Key Points

- In a large university hospital in Lyon, France, the accuracy, sensitivity, specificity, PPV, and NPV of chest CT for COVID-19 reached $88.9 \%, 90.2 \%, 88 \%, 84.1 \%$, and $92.7 \%$, respectively, using RT-PCR as standard of reference.
\end{abstract}

Salim Si-Mohamed

salim.si-mohamed@chu-lyon.fr

1 Department of Radiology, Hospices Civils de Lyon, Lyon, France

2 PHILIPS France, 33, rue de Verdun, CS 60 055, 92156 Suresnes Cedex, France

3 Laboratoire de Virologie, Institut des Agents Infectieux de Lyon, Centre National de Référence des virus respiratoires France Sud, Centre de Biologie et de Pathologie Nord, Hospices Civils de Lyon, F-69317 Lyon, France

4 Université de Lyon, Virpath, CIRI, INSERM U1111, CNRS UMR5308, ENS Lyon, Université Claude Bernard Lyon 1, F-69372 Lyon, France
5 Univ Lyon, INSA-Lyon, Université Claude Bernard Lyon 1, UJM-Saint Etienne, CNRS, Inserm, CREATIS UMR 5220, U1206, F-69621 Lyon, France

6 Department of cardiothoracic and vascular imaging, $\mathrm{CHU}$ cardiologique Louis Pradel, 59 Boulevard Pinel, 69500 Bron, France

7 Department of Emergency, Croix-Rousse Hospital, Hospices Civils de Lyon, Lyon, France

8 Department of Information Technology, Hospices Civils de Lyon, Lyon, France

9 Service de Maladies Infectieuses et Tropicales, Hôpital Croix-Rousse, Hospices Civils de Lyon, Lyon, France

10 LabTAU INSERM U1032, Lyon, France

11 Service de Biostatistique et Bioinformatique des HCL, Laboratoire Biostatistique Santé, UMR 5558, 69424 Lyon, France 


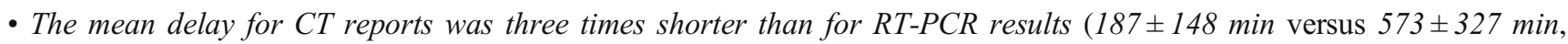
$p<0.0001)$.

- Due to high accuracy of chest CT for COVID-19 and shorter time for CT reports than RT-PCR results, chest CT can be used to orient patients suspected to be positive towards the COVID+ unit to decrease congestion in the emergency departments.

Keywords Coronavirus $\cdot$ Emergency medicine $\cdot$ Tomography, X-ray $\cdot$ Diagnostic imaging $\cdot$ Sensitivity and specificity

\begin{tabular}{ll}
\multicolumn{2}{l}{ Abbreviations } \\
COVID-19 & Coronavirus disease 2019 \\
CT & Computed tomography \\
HCL & Hospices Civils de Lyon \\
NPV & Negative predictive value \\
PPV & Positive predictive value \\
RT-PCR & $\begin{array}{l}\text { Reverse transcription polymerase } \\
\text { chain reaction }\end{array}$
\end{tabular}

Introduction

As COVID-19 spreads around the world, it causes an influx in the emergency departments of the hospitals $[1,2]$. In this context, quick triage of patients towards the COVID+ and COVID- hospital units is essential.

There is a growing interest in the role and appropriateness of chest computed tomography (CT) for the screening, diagnosis, and management of patients with suspected COVID-19 infection. This interest is reinforced taking into account the limited availability of virological testing kits to date, the delays concerning the output of the virological test, the concern for test sensitivity from earlier reports in China, and the growing number of publications describing the $\mathrm{CT}$ appearance in the setting of known or suspected COVID-19 infection [3-7].

In our institution, we opted to carry out chest $\mathrm{CT}$ as well as virological tests in any patient in multiple emergency departments, for which a hospitalization was planned whatever the reasons, i.e., COVID- or non-COVID-related, in order to orient those patients in COVID+ or COVID- units.

The goal of this study was to analyze the diagnostic performances of the chest CT for COVID-19 compared with reverse transcription polymerase chain reaction (RT-PCR) as method of reference, and to compare the delay in reporting results between the two methods, before orienting the patients towards the appropriate hospital unit.

\section{Material and methods}

\section{Population}

This multi-departmental monocentric retrospective study was conducted in a large university hospital (Hospices Civils de Lyon (HCL), Lyon, France) from March 3 to April 4, 2020.
All CT examinations requested by three general emergency departments of the HCL (Hospital Croix-Rousse, Hospital Edouard Herriot, Hospital Lyon Sud) during this period were extracted from our Radiology Electronic Requesting system. Patients under 18 years old were excluded. A total of 1945 CTs were requested, including 720 chest CTs among which 694 were indicated for COVID-19 detection. All these 694 patients (sex-ratio $\mathrm{M} / \mathrm{F}=363 / 331$, mean age $=66.4 \pm 18.63$ years) required a hospitalization based on their clinical symptoms at admission. They all underwent a virological test. The main reasons for admission at the emergency department were classified as follows: pulmonary and otolaryngology, abdominal, syncopal episode, trauma, deterioration of general condition, confusion-coma, stroke, and psychiatric. Presence of dyspnea, caught, rhinitis, throat, and/or chest pain, fever (defined as a temperature above $38^{\circ} \mathrm{C}$ ), and anosmia were recorded. Patients with none of these signs were classified as "asymptomatic." Data usage policy of our institution in terms of confidentiality, anonymization, and security was applied for each study and approval was obtained from the ethical committee.

\section{CT protocols and analysis}

Chest CTs were performed on different helical CT systems, including Revolution GSI ${ }^{\circledR}$ (GE Healthcare), iCT 256® (Philips Healthcare), Ingenuity CT® (Philips Healthcare), SOMATOM Definition AS ${ }^{\circledR}$ (Siemens Healthineers), SOMATOM Definition AS+® (Siemens Healthineers), and Aquilion Lightning ${ }^{\circledR}$ (Canon Medical Systems). The scanning parameters were as follows: mean tube voltage of $119.3 \pm 11.9 \mathrm{kVp}$ (range: $80-140 \mathrm{kVp}$ ), mean slice thickness of $2.3 \pm 0.8 \mathrm{~mm}$ (range: $0.6-3 \mathrm{~mm}$ ). Scan length, mAs, and field of view were adapted for each patient and clinical indication, including exploration of the abdomen when required. One hundred and twenty-two (122) out of the six hundred and ninety-four (694) examinations (17.6\%) were obtained after intravenous administration of iodinated contrast material. The purpose for injection was the detection of a pulmonary embolism in 105 patients and thoracic-abdominal and pelvic exploration in 17 patients. All acquisitions were performed at the end of a deep inspiration.

All the chest CTs were analyzed as part of the clinical workflow by senior radiologists from the HCL, including during night shift. The presence of typical features of COVID disease were identified, including ground glass opacity 
(GGO), crazy paving, sub-pleural bands of consolidations, reversed halo sign, and lung consolidations as described previously [3, 8-12]. An example of these signs is shown in Fig. 1. On the final report, patients were rated as "Surely COVID+" when presenting with peripheral, bilateral, or multifocal GGO of rounded morphology \pm consolidation or crazy paving, reversed halo sign, or sub-pleural bands of consolidations. Patients were rated as "Possible COVID+" when presenting with multifocal, diffuse, peripheral, or unilateral GGO \pm consolidation lacking a specific distribution and nonrounded or non-peripheral or with only few very small GGO with a non-rounded and non-peripheral distribution or with atypical findings: large pleural effusion, major lymph node size increase, or bronchiolitis pattern. Patients were rated as "COVID-" when the chest CT was normal or demonstrating another pathology.

In order to assess the inter-reader reproducibility of CT scan analysis, 70 randomly selected patients $(10 \%$ of the total dataset) were re-analyzed by an experienced senior radiologist (15 years of experience in the thoracic imaging field).
The delay between the chest CT request by the emergency department and the validation of the report by the radiologist was recorded for all patients. We also recorded whether the CT was performed during day or night shift.

\section{Virological tests}

All patients were tested for SARS-CoV-2 nucleic acid detection by RT-PCR assay on upper and/or lower respiratory samples. Clinical specimens for 2019-nCoV diagnostic testing were obtained in accordance with the World Health Organization guidelines (WHO/COVID-19/laboratory/ 2020.5). The RT-PCR assays were performed using the RdRp IP2-IP4 primers and probes of the Institut Pasteur protocol, which is used in France for SARS-CoV-2 detection by the National Reference Center for Respiratory Virus Infection and French hospital laboratories [13]. This protocol, detecting two targets in RdRp gene, was adapted on the Panther Fusion ${ }^{\circledR}$ (Hologic) molecular system in the Laboratory of Virology (Institut des Agents Infectieux) of the HLC, for high
Fig. 1 Typical images from five different patients COVID-19 positive at virology test: ground glass opacity (a), crazy paving (b), posterior lung consolidations (c) and sub-pleural bands of consolidations (d), reversed halo sign (e)

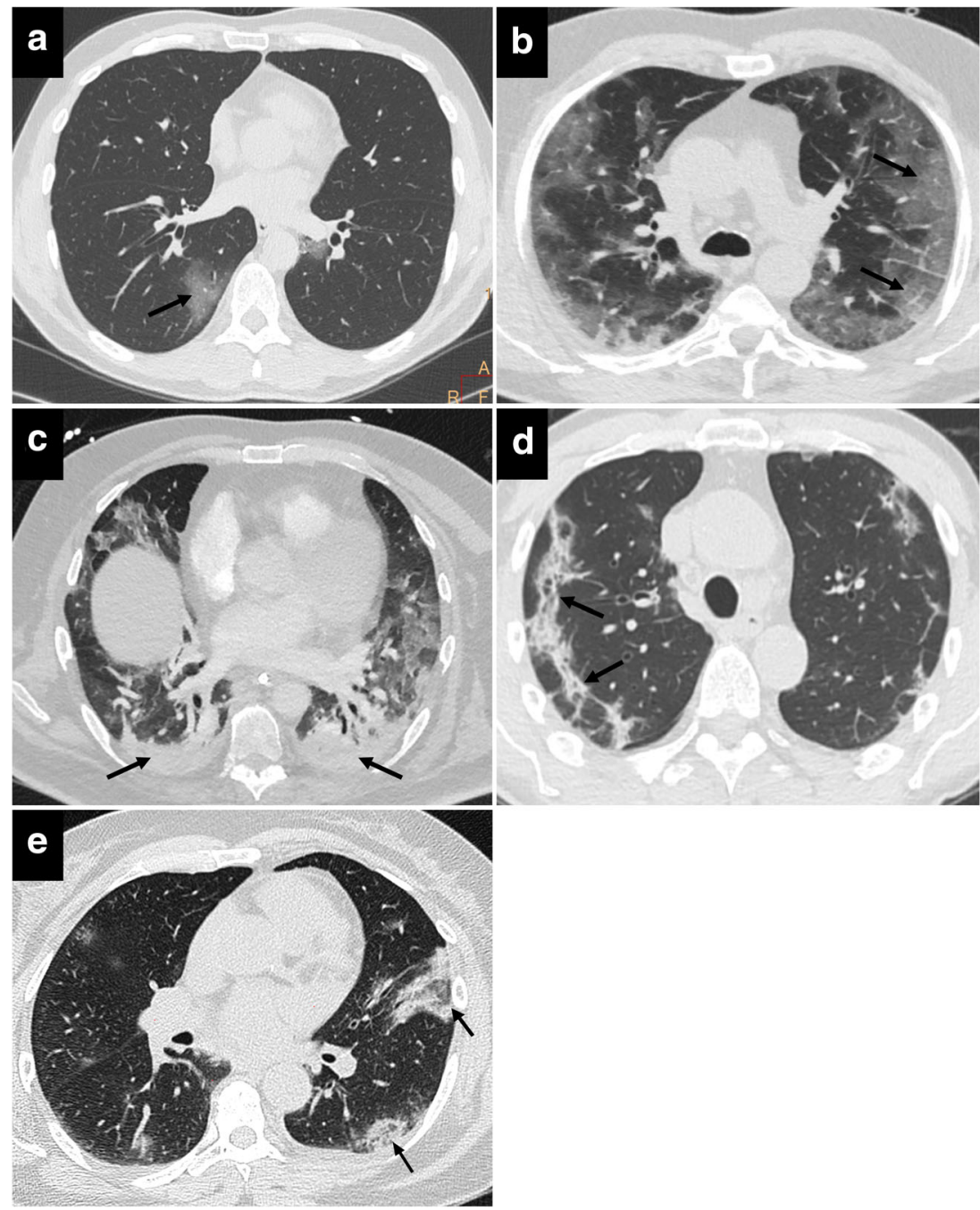


throughput diagnostic. Two hundred microliters of sample was transferred in the Hologic Panther lysis buffer tube $(710 \mu \mathrm{L})$ in BSL2 biosafety conditions and then loaded in the machine for nucleic acid extraction and molecular amplification according to the manufacturer's protocol. The quality effectiveness of the process was checked using an internal control tested for each sample. All amplification curves were checked before validation. RT-PCR tests were all performed independently of the $\mathrm{CT}$ findings.

The result of the RT-PCR testing of the first sample was recorded for all patients and was classified as "COVID+" or "COVID-" initial virology diagnostic. In case of multiple samples, in the days following the initial test, the final virological diagnostic was rated as COVID+ if one of the samples was positive.

The delay between the RT-PCR test request by the emergency department and the validation of the report by the virologist was recorded for all patients. It takes in account, the delivery time of the sample from three different hospitals to one lab by shuttle every $1.5 \mathrm{~h}$, the record and the treatment of the sample before analysis. The sample molecular analysis is 2.5-h long and was done, at the time of the study, from 8:30 am to 9:00 pm but not all night long.

\section{Statistical analysis}

All statistics were performed using R Statistical Software (Foundation for Statistical Computing, version 3.6.1).
Quantitative variables were described as mean \pm standard deviation and ranges. Accuracy, sensitivity, specificity, positive predictive value (PPV), and negative predictive value (NPV) were estimated for "Surely COVID+" class as positive CT and "Possible COVID+" and "COVID-" CT classes as negative CT on one hand and "Surely COVID+" and "Possible COVID+" classes as positive CT and "COVID-" class as negative $\mathrm{CT}$ on the other hand, both compared with the final RT-PCR results considered as the gold standard. Ninety-five percent confidence intervals were built using the Wilson's method. Agreement between the CT and the virological diagnostic was measured using kappa coefficient. Inter-reader reproducibility between the initial $\mathrm{CT}$ analysis and the analysis from the experienced radiologist on the 70 randomly selected patients was measured using the kappa coefficient.

Differences in the delays between $\mathrm{CT}$ examination and virology report were compared with the paired Student $t$ test.

\section{Results}

Chest $\mathrm{CT}$ and initial virology test results were available for the 694 patients. A flowchart of the study is shown in Fig. 2. Main reasons for admittance together with the COVID-19 relevant symptoms are provided in Table 1. Pulmonary and otolaryngology symptomatology was the main reason for admission at the emergency department for $67 \%$ of the patients.

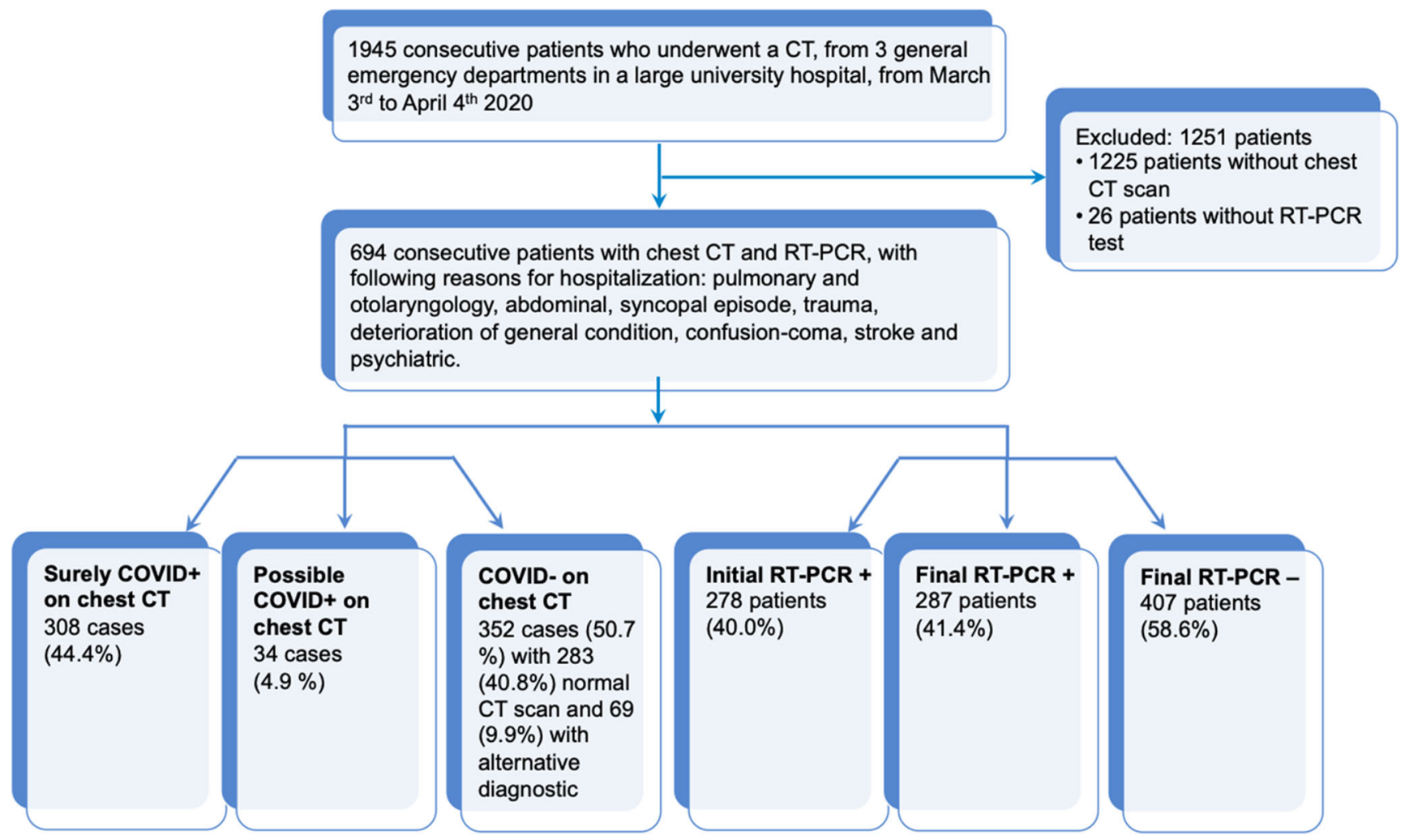

Fig. 2 Flowchart of the study 
Table 1 Main reasons for admittance at the emergency department by clinical category and COVID-19 relevant symptoms per category

Main reasons for admittance with COVID-19-related

symptoms per category

Pulmonary and otolaryngology

Dyspnea

Caught, rhinitis, throat/chest pain

Fever

Anosmia

Asymptomatic

Abdominal

Dyspnea

Caught, rhinitis, throat/chest pain

Fever

Anosmia

Asymptomatic

Trauma

Dyspnea

Caught, rhinitis, throat/chest pain

Fever

Anosmia

Asymptomatic

Syncopal episode

Dyspnea

Caught, rhinitis, throat/chest pain

Fever

Anosmia

Asymptomatic

Deterioration of general condition

Dyspnea

Caught, rhinitis, throat/chest pain

Fever

Anosmia

Asymptomatic

Confusion-coma

Dyspnea

Caught, rhinitis, throat/chest pain

Fever

Anosmia

Asymptomatic

Psychiatric

Dyspnea

Caught, rhinitis, throat/chest pain

Fever

Anosmia

Asymptomatic

Stroke

Dyspnea

Caught, rhinitis, throat/chest pain

Fever

Anosmia

Asymptomatic

Others

Dyspnea

Caught, rhinitis, throat/chest pain

Fever

Anosmia

Asymptomatic
Number of

patients $(n=694)$

$\begin{array}{rr}465(67.0 \%) & 228 \\ 377 & 187 \\ 361 & 183 \\ 267 & 166 \\ 71 & 51 \\ 1 & 0\end{array}$

$4(10.7 \%)$

10

21

28

5

33

$51(7.3 \%)$

6

8

7

0

39

$24(3.4 \%)$

4

6

7

1

11

$20(2.9 \%)$

2

7

7

0

9

$18(2.6 \%)$

4

3

3

0

10

$6(0.9 \%)$

2

1

1

0

3

$11(1.6 \%)$

1

2

2

0

7

$25(3.6 \%)$

1

5

10

0

11
Number of RT-PCR

positive patients $(n=287)$

$228(49.0 \%)$

187

183

166

51

$21(28.4 \%)$

5

10

11

5

5

$9(17.6 \%)$

2

4

1

0

5

$8(33.3 \%)$

3

3

3

0

2

$6(30.0 \%)$

1

4

4

0

$6(33.3 \%)$

3

0

0

3

$1(16.7 \%)$

0

1

1

0

0

$6(54.5 \%)$

0

1

2

0

4

$2(8.0 \%)$

0

0

1

0

1 
Rate of RT-PCR positive tests varied with regard to the reason for admission and was relatively high even for nonCOVID-19-related symptoms such as trauma (17.6\% which corresponds to 9 patients among which 5 were completely asymptomatic with a positive RT-PCR) or psychiatric disorders $(16.6 \%)$. It is noticeable that among the 694 patients, 124 patients $(17.9 \%)$ were fully asymptomatic to COVID common signs from which 21 (17\%) had RT-PCR positive tests.

Regarding initial RT-PCR, sampling site was the upper respiratory tract (nasopharyngeal swabs or aspirates) in 685 patients and the lower respiratory tract (endotracheal aspirate or bronchoalveolar lavage) in 9 patients (positive in 3 cases). Additionally, 20 patients underwent multiple RT-PCR during their hospital stay ( 2 tests in 19 patients and 4 tests in one patient). Sampling site was the upper respiratory tract in 18 patients and the lower respiratory tract in two patients (both negative). For these 20 patients, RT-PCR has been repeated as the patient was negative at the first test with an imperative need of knowing his COVID status for optimal care adaptation or hospital organizational reasons (14 patients were in intensive care unit, 5 had surgery, and one was transferred to a dialysis center). All the patients were initially scheduled to be hospitalized but 54 patients $(7.8 \%)$ were finally discharged.

On the initial RT-PCR test, 278 patients were tested positive to COVID-19 and 287 were positive on the final RT-PCR test, leading to a final prevalence of the disease of $41.4 \%$.

Chest CTs were rated as "Surely COVID+" in 308 cases (44.4\%), "Possible COVID+" in 34 cases $(4.9 \%)$, and "COVID-" in 352 cases (50.7\%), including 283 (40.8\%) normal chest CTs and 69 (9.9\%) chest CTs demonstrating another pathology than COVID-19.

Using the final virological diagnosis as standard of reference, when considering the "Surely COVID+" CT class as positive CT and "Possible COVID+" and "COVID-" CT classes as negative CT, chest CT accuracy was calculated at $88.9 \%$ (95\% CI 86.4-90.0\%), sensitivity at 90.2\% (95\% CI 87.3-93.2\%), specificity at 88\% (95\% CI 84.4-90.8\%), PPV at $84.1 \%(95 \%$ CI $79.6-87.8 \%)$, and NPV at $92.7 \%(95 \% \mathrm{CI}$
89.7-94.9\%) (Table 2). Agreement between the two methods was good with a kappa of 0.77 (95\% CI 0.73-0.82).

Similarly, when considering the "Surely COVID+" and "Possible COVID+" classes as positive CT and "COVID-" class as negative $\mathrm{CT}$, chest $\mathrm{CT}$ accuracy was calculated at $86.6 \%$ (95\% CI 83.9-88.9\%), sensitivity at $93.4 \%$ (95\% CI $89.9-95.7 \%)$, specificity at $81.8 \%$ (95\% CI $77.8-85.3 \%)$, PPV at $78.4 \%(95 \%$ CI $73.7-82.4 \%)$, and NPV at $94.6 \%$ (95\% CI 91.7-96.5\%) (Table 2). Agreement between the two methods was good with a kappa of 0.73 (95\% CI 0.68 0.78).

Radiologists were aware of the results of the RT-PCR when doing the report in 18 patients including 6 patients positive to COVID-19 and 12 negative. Three hundred and six (44\%) of the CT scans were performed during night shift (6:30 PM to 7:30 AM). Inter-reader reproducibility was very good with a kappa of 0.86 . Mean volumetric Computed Tomography Dose Index (CTDIvol) and mean Dose Length Product (DLP) were 9.71 \pm 4.39 mGy (range: $2.8-33.9$ mGy) and $387.4 \pm 223.6$ mGy.cm (range: 104.9-1903 mGy.cm) respectively.

The mean delay between the examination request by the emergency department and the validation of the report was $187 \pm 148 \mathrm{~min}$ (range: $21-1267 \mathrm{~min}$, median: $154 \mathrm{~min}$, interquartile range: $138 \mathrm{~min}$ ) and $573 \pm 327 \mathrm{~min}$ (range: 189 $2812 \mathrm{~min}$, median: $444 \mathrm{~min}$, interquartile range: $403 \mathrm{~min}$ ) for chest CT and RT-PCR respectively $(p<0.0001)$.

\section{Discussion}

Since the beginning of the COVID-19 epidemic phase, finding ways to control the congestion in emergency departments has been of major importance. As such, it is imperative to have a reliable and rapid tool for correctly referring patients to COVID+ or COVID- hospital units. Virological tests by nasopharyngeal sampling have been widely used for this task, but the relative reliability of upper respiratory virus detection
Table 2 Diagnostic performance of the chest CT for the "Surely COVID+" rated CT examinations (column 1) and the "Surely COVID+" and "Possible COVID+" rated CT examinations together (column 2) with final RT-PCR results as gold standard. 95\% confidence intervals are provided in brackets

\begin{tabular}{lll}
\hline & $\begin{array}{c}\text { "Surely COVID+" } \\
\text { CT vs RT-PCR }\end{array}$ & $\begin{array}{l}\text { "Surely COVID+" } \\
\text { and "Possible COVID+" } \\
\text { CT vs RT-PCR }\end{array}$ \\
\hline True positive & 259 & 268 \\
True negative & 358 & 333 \\
False positive & 49 & 74 \\
False negative & 28 & 19 \\
Accuracy & $88.9 \%(86.4-90.0)$ & $86.6 \%(83.9-88.9 \%)$ \\
Sensitivity & $90.2 \%(87.3-93.2)$ & $93.4 \%(89.9-95.7 \%)$ \\
Specificity & $88 \%(84.4-90.8)$ & $81.8 \%(77.8-85.3 \%)$ \\
Positive predictive value & $84.1 \%(79.6-87.8)$ & $78.4 \%(73.7-82.4 \%)$ \\
Negative predictive value & $92.7 \%(89.7-94.9)$ & $94.6 \%(91.7-96.5 \%)$ \\
\hline
\end{tabular}


and the delays in obtaining results prompt to find alternative solutions, mainly by using chest CT.

In our study, when considering only the "Surely COVID+" chest CT, accuracy, when compared with the final results of the virological tests, reached $88.9 \%$ with a sensitivity and specificity of $90.2 \%$ and $88 \%$ respectively. In previously published results, sensitivity and specificity varied from 60 to $98 \%$ and 25 to $53 \%$ respectively $[4-7,14,15]$. These variabilities concerning the values of sensitivity and specificity are most likely due to the retrospective nature of these studies, including ours, but also to differences in virological test accuracy. In our study, specificity is particularly higher than other studies, probably because alternative diagnosis to COVID-19 was relatively infrequent mainly since flu and other respiratory virus epidemic were ending in France which may not be the case for the other studies that occurred earlier in the year. Indeed, even if some differences have been reported between the CT presentation of flu and COVID, the differences remain small and difficult to assess in clinical routine [16]. Furthermore, specificity is probably underestimated in most of the studies, including ours, since the sensitivity of virological tests is questionable which leads to erroneous false positives in CT. Indeed, besides the performance of the detection kits themselves, which is high and comparable between the different tests available on the market (including ours) [17, 18], several external factors may affect RT-PCR detectability including sampling operations, specimens source (upper or lower respiratory tract), sampling timing (delay after the illness onset), and performance of detection kits [19]. Upper respiratory tract sample, representing the majority of the samples in our study (98.5\%), has been reported to have a detection rate comprising between $16.4 \%$ [20] and 63\% [16] for the nasal swabs, lowermost than lower respiratory tract samples such as sputum (66.4\% [20] to 72\% [16]) or bronchoalveolar lavage (93\%) [16]. We should also take into account that the detectable viral load depends on the days after the illness onset and the site sampled. In the first 5-6 days after onset, SARS$\mathrm{CoV}-2$ could most reliably be detected in the nasopharyngeal tract $[20,21]$. The peak of viral shedding is reported at 56 days after onset $[19,20]$. Seven to 9 days after onset, sputum is more reliable than nasopharyngeal swabs for viral detection $[20,22]$. Other studies have also shown that the detection of the virus from a sputum is of a significantly longer period of time compared with the nasopharyngeal swab (29 days versus 11 days) [19]. Given the variability in the viral loads according to the time and the site of sampling, a negative test result from respiratory samples does not rule out the disease [23]. In contrary to the CT specificity which suffers from the imperfect RT-PCR sensitivity as mentioned above, the CT sensitivity is surely more reliable as specificity of RT-PCR is reported to be very high [13].

Regarding the PPV and NPV, Wen et al [7] reported a PPV and NPV of $92 \%$ and $42 \%$ respectively. In our study, we found a PPV of $84.1 \%$ and a NPV of $92.7 \%$. Same as for the calculated specificity, the false negatives of the virological tests probably decreased the apparent PPV of chest CT. High NPV can be explained by the population taking part in this study, mostly patients with severe illness since they were scheduled for hospitalization and thus with a low probability of having a false negative chest CT. Furthermore, the difference in prevalence of the disease between our study and the study of Wen et al [7] (41.4\% versus $85 \%$ ) partially explains the difference in NPV.

Considering the "Possible COVID+" CT results as positive CT increased the sensitivity (from 90.2 to $93.4 \%$ ) and the NPV (from 92.7 to $94.6 \%$ ) but impaired the overall accuracy (from 88.9 to $86.6 \%$ ), the specificity (from $88 \%$ to $81.8 \%$ ) and the PPV (from 84.1 to $78.4 \%$ ) of chest CT, due to the inclusion of atypical CT patterns. In our study, the clinical objective was to refer quickly the positive patients to the COVID+ units. As a result, the inclusion of "Possible COVID+" diagnosis was not suitable since the PPV was decreased. Conversely, after the pandemic phase, the better NPV might lead to include these "Possible COVID+" diagnosis in order to benefit from the good NPV to safely address the negative patients to the COVID- units. When this pandemic phase will be over, the management of CT COVID+ patients will have to integrate the prevalence of other pulmonary diseases with CT findings equivalent to those of COVID-19 such as influenza. This may lead to isolate these patients in the emergency department until the RT-PCR results are available.

Regarding the delay to obtain the results for a given patient, RT-PCR results, obtained with high throughput diagnostic system, were available after in average $9.5 \mathrm{~h}$ (median: $7.4 \mathrm{~h}$ ). Even if real time RT-PCR is the fastest available viral detection method, chest CT clearly outperforms the RT-PCR in terms of delay to report the presence of COVID-19 infection. In our study, the delay between the request by the emergency department and the validation of the report was three times longer for the virological test than for chest CT.

Taking into account the difference in delays and the NPV and PPV aforementioned values, we can consider the possibility to make use of the chest CT to orient patients suspected to be positive towards the COVID+ unit to decrease congestion in the emergency departments. In the current epidemic phase, suspected positive patients can then wait, in an isolated room, in the COVID+ unit for the final virological tests. As the NPV is not equal to $100 \%$, we consider that the probability of orienting a positive patient towards the COVID- unit is high and the risk of contaminating other patients is important. As a result, for patients that are negative according to chest $\mathrm{CT}$, we suggest that they wait in the emergency department until the results of the virological test are available and then be oriented towards to the COVID- or COVID+ units accordingly. The interest of this systematic strategy is reinforced by the fact that, during the epidemic phase, a high rate of RT- 
PCR COVID-19 positive patients was observed in patients with non-COVID-19-related symptoms such as trauma $(17.6 \%)$ or psychiatric disorder $(16.6 \%)$.

There are several limitations in the present study. First as discussed before, by using RT-PCR assays with relatively low positive rate in upper respiratory samples as reference, the PPV and the specificity of chest CT for COVID-19 may be underestimated. A second limitation is that access to serology data that might help to reclassify RT-PCR false negatives was not possible at the moment of the study and is difficult to perform retrospectively. Furthermore, the relative sensitivity and specificity of these tests might not allow fully solving the problem [24]. A third one is that in the current pandemic context, a positive RT-PCR does not necessarily imply that there is SARS-CoV-2 pneumonia. A fourth one is related to the difficulties in generalizing our results in areas or periods of time with lower disease prevalence. Indeed, PPV would certainly be decreased in these settings. Nevertheless, the overload of the emergency departments that we observed during the epidemic peak would also be less important and as a result the strategy of triage should be adapted accordingly. An additional limitation concerns the retrospective nature of the study with the common bias related to this kind of studies. Nevertheless, we are confident that this bias risk is limited as the information system of our institution allowed to take into account all the patients going into the emergency department and all the requested CT scans. Finally, we should mention that we did not perform a sample size calculation prior to our study. Indeed, our study is a retrospective study in which we took into account all the patients matching the inclusion criteria since beginning of March 2020.

\section{Conclusion}

During the COVID-19 epidemic phase, in a high prevalence environment of the disease, chest $\mathrm{CT}$ is a rapid and most probably an adequately reliable triage tool to refer patients requiring hospitalization to the COVID+ or COVID- hospital units, when response times for virological tests are too long, in order to decrease congestion in the emergency departments.

Acknowledgments We acknowledge Morgane Bouin, Cécile Rémy, Hind Behlouli, and Sabine Debeer for their help in data collection.

Funding information The authors state that this work has not received any funding.

\section{Compliance with ethical standards}

Guarantor The scientific guarantor of this publication is Pr. Loic Boussel.
Conflict of interest Anna Vlachomitrou declares relationships with the following companies: Philips.

Statistics and biometry Murielle Rabilloud kindly provided statistical advice for this manuscript.

Informed consent Written informed consent was waived by the Institutional Review Board.

Ethical approval Institutional Review Board approval was obtained.

Methodology
- retrospective
- diagnostic study
- multicenter study

\section{References}

1. Mareiniss DP (2020) The impending storm: COVID-19, pandemics and our overwhelmed emergency departments. Am J Emerg Med. https://doi.org/10.1016/j.ajem.2020.03.033

2. Mitchell R, Banks C, authoring working party (2020) Emergency departments and the COVID-19 pandemic: making the most of limited resources. Emerg Med J. https://doi.org/10.1136/ emermed-2020-209660

3. Bai HX, Hsieh B, Xiong Z et al (2020) Performance of radiologists in differentiating COVID-19 from viral pneumonia on chest CT. Radiology 200823. https://doi.org/10.1148/radiol.2020200823

4. Ai T, Yang Z, Hou H et al (2020) Correlation of chest CT and RTPCR testing in coronavirus disease 2019 (COVID-19) in China: a report of 1014 cases. Radiology 200642. https://doi.org/10.1148/ radiol.2020200642

5. Fang Y, Zhang H, Xie J et al (2020) Sensitivity of chest CT for COVID-19: comparison to RT-PCR. Radiology 200432. https:// doi.org/10.1148/radiol.2020200432

6. Simpson S, Kay FU, Abbara S et al (2020) Radiological Society of North America expert consensus statement on reporting chest CT findings related to COVID-19. Endorsed by the Society of Thoracic Radiology, the American College of Radiology, and RSNA. Radiology Cardiothoracic Imaging 2:e200152. https://doi.org/10. 1148/ryct.2020200152

7. Wen Z, Chi Y, Zhang L et al (2020) Coronavirus disease 2019: initial detection on chest ct in a retrospective multicenter study of 103 Chinese subjects. Radiology Cardiothoracic Imaging 2: e200092. https://doi.org/10.1148/ryct.2020200092

8. Wang H, Wei R, Rao G, Zhu J, Song B (2020) Characteristic CT findings distinguishing 2019 novel coronavirus disease (COVID19) from influenza pneumonia. Eur Radiol. https://doi.org/10.1007/ s00330-020-06880-Z

9. Revel M-P, Parkar AP, Prosch H et al (2020) COVID-19 patients and the radiology department - advice from the European Society of Radiology (ESR) and the European Society of Thoracic Imaging (ESTI). Eur Radiol. https://doi.org/10.1007/s00330-020-06865-y

10. Yang W, Sirajuddin A, Zhang X et al (2020) The role of imaging in 2019 novel coronavirus pneumonia (COVID-19). Eur Radiol. https://doi.org/10.1007/s00330-020-06827-4

11. Ye Z, Zhang Y, Wang Y, Huang Z, Song B (2020) Chest CT manifestations of new coronavirus disease 2019 (COVID-19): a pictorial review. Eur Radiol. https://doi.org/10.1007/s00330-02006801-0

12. Zhou Z, Guo D, Li C et al (2020) Coronavirus disease 2019: initial chest CT findings. Eur Radiol. https://doi.org/10.1007/s00330-02006816-7 
13. World Health Organization (2020) Protocol: real-time RT-PCR assays for the detection of SARS-CoV-2, Institut Pasteur, Paris. World Health Organization, Geneva. Available via https://www. who.int/docs/default-source/coronaviruse/real-time-rt-pcr-assaysfor-the-detection-of-sars-cov-2-institut-pasteur-paris.pdf?sfvrsn= 3662fcb6_2. Accessed 11 Jan 2020

14. Long $\mathrm{C}, \mathrm{Xu} \mathrm{H}$, Shen Q et al (2020) Diagnosis of the coronavirus disease (COVID-19): rRT-PCR or CT? Eur J Radiol 126:108961. https://doi.org/10.1016/j.ejrad.2020.108961

15. Inui S, Fujikawa A, Jitsu M et al (2020) Chest CT findings in cases from the cruise ship "diamond princess" with coronavirus disease 2019 (COVID-19). Radiol Cardiothorac Imaging 2:e200110. https://doi.org/10.1148/ryct.2020200110

16. Wang W, Xu Y, Gao R et al (2020) Detection of SARS-CoV-2 in different types of clinical specimens. JAMA. https://doi.org/10. 1001/jama.2020.3786

17. Sethuraman N, Jeremiah SS, Ryo A (2020) Interpreting diagnostic tests for SARS-CoV-2. JAMA. https://doi.org/10.1001/jama.2020. 8259

18. Nalla AK, Casto AM, Huang M-LW et al (2020) Comparative performance of SARS-CoV-2 detection assays using seven different primer-probe sets and one assay kit. J Clin Microbiol 58. https:// doi.org/10.1128/JCM.00557-20
19. Chen C, Gao G, Xu Y et al (2020) SARS-CoV-2-positive sputum and feces after conversion of pharyngeal samples in patients with COVID-19. Ann Intern Med. https://doi.org/10.7326/M20-0991

20. Yu F, Yan L, Wang N et al (2020) Quantitative detection and viral load analysis of SARS-CoV-2 in infected patients. Clin Infect Dis. https://doi.org/10.1093/cid/ciaa345

21. Wölfel R, Corman VM, Guggemos W et al (2020) Virological assessment of hospitalized patients with COVID-2019. Nature. https://doi.org/10.1038/s41586-020-2196-x

22. Lescure F-X, Bouadma L, Nguyen D et al (2020) Clinical and virological data of the first cases of COVID-19 in Europe: a case series. Lancet Infect Dis. https://doi.org/10.1016/S1473-3099(20) 30200-0

23. Winichakoon P, Chaiwarith R, Liwsrisakun C et al (2020) Negative nasopharyngeal and oropharyngeal swabs do not rule out COVID19. J Clin Microbiol 58. https://doi.org/10.1128/JCM.00297-20

24. Nuccetelli M, Pieri M, Grelli S et al (2020) SARS-CoV-2 infection serology: a useful tool to overcome lockdown? Cell Death Dis 6:38. https://doi.org/10.1038/s41420-020-0275-2

Publisher's note Springer Nature remains neutral with regard to jurisdictional claims in published maps and institutional affiliations. 